\title{
Untangling mixed modes in model-independent analysis of beam dynamics in circular accelerators
}

\author{
Chun-xi Wang* \\ Argonne National Laboratory, 9700 South Cass Avenue, Argonne, Illinois 60439, USA
}

(Received 18 December 2003; published 17 November 2004)

\begin{abstract}
Model-independent analysis of beam dynamics in circular accelerators reveals spatial-temporal modes due to beam oscillations. Although such modes have been shown to be informative, their usefulness is limited by the lack of quantitative understanding in general. Here we present a simple approach to studying the modes analytically, which results in a quantitative understanding of the modes and an algorithm to extract the physical modes by untangling the mixed singular value decomposition modes. Particularly, we focus on the coupled betatron modes that are of great interest for high-energy colliders. A simple relationship between the coupling modes and the lattice functions is established, which not only provides a quantitative understanding but also lays the foundation for using these modes to measure machine properties and beam motion in phase space.
\end{abstract}

DOI: 10.1103/PhysRevSTAB.7.114001

PACS numbers: 29.27.Bd, 29.20.Dh, 41.75.-i

\section{INTRODUCTION}

In recent years, model-independent analysis (MIA) has emerged as a new approach to studying beam dynamics by analyzing simultaneously recorded beam histories at a large number of beam position monitors (BPMs) [1,2]. A basic technique used in MIA is the spatial-temporal mode analysis via a singular value decomposition (SVD) of the data matrix containing beam histories. Similar to Fourier analysis, SVD mode analysis decomposes the spatial-temporal variation of the beam centroid into superpositions of various orthogonal modes by effectively accomplishing a major statistical data analysis, namely, the principal component analysis $[3,4]$. Let $b_{p}^{m}$ represent the measurement at the $m$ th monitor for the $p$ th pulse or turn. Then the singular value decomposition of the beam-history matrix $B_{P \times M}=\left(b_{p}^{m}\right)$ gives

$$
B=\hat{U} S \hat{V}^{T}=\sum_{\text {modes }} \sigma_{i} u_{i} v_{i}^{T}
$$

where $\hat{U}_{P \times P}=\left[u_{1}, \ldots, u_{P}\right]$ and $\hat{V}_{M \times M}=\left[v_{1}, \ldots, v_{M}\right]$ are orthonormal matrices comprising the eigenvectors, and $S_{P \times M}$ is a diagonal matrix with non-negative singular values $\sigma_{i}$ along the upper diagonal. Preferably $P \gg M$. Since the $u$ vectors depict turn-by-turn time variations and the $v$ vectors depict spatial variation along BPMs, they are referred to as temporal and spatial vectors, respectively. A pair of spatial and temporal vectors characterizes an eigenmode and the corresponding singular value specifies its strength.

Given the measured BPM data, it is easy to numerically compute the SVD modes, which have been shown to reveal valuable information in beam dynamics studies [1,2,5-14]. However, most of our understanding of these

\footnotetext{
*Electronic address: wangcx@aps.anl.gov
}

modes was rather qualitative and thus limited the modes' usefulness, especially due to the fact that the orthogonal SVD modes could be a mix of several nonorthogonal physical modes. A remarkable work-around was developed for lattice validation $[6,7,10]$ where Green-functiontype transfer-matrix elements are derived from any set of independent orbits, such as the spatial vectors of the betatron modes.

For a better understanding of the SVD modes, it is necessary to establish the relationship between the SVD modes and the underlying physics by analytically solving the SVD problem. This has been done only for betatron motion in 1 degree of freedom, and the result allows accurate measurement of phase advances and beta function in storage rings with little coupling [14]. A desirable extension (especially for colliders) is to take into account the transverse coupling and dispersion, which requires solving the nontrivial SVD problem in the phase space of $2 \frac{1}{2}$ degrees of freedom. Unfortunately, it seemed hopeless to extend the approach used in [14], i.e., to solve the eigensystem $B^{T} B v=\sigma^{2} v$ with $\left(B^{T} B\right)_{m n}=\bar{J}_{a} \sqrt{\beta_{a}^{m} \beta_{a}^{n}} \gamma^{m} \gamma^{n} \cos \left(\psi_{a}^{m}-\right.$ $\left.\psi_{a}^{n}\right)+\bar{J}_{b} \sqrt{\beta_{a}^{m} \beta_{a}^{n}} c_{b}^{m} c_{b}^{n} \cos \left(\psi_{b}^{m}-\psi_{b}^{n}+\Delta \psi_{b}^{m}-\Delta \psi_{b}^{n}\right)$ to include transverse coupling, where the various quantities will be explained shortly. It turns out that there is a simple approach to overcome this obstacle and reveal the relationship between the SVD modes and the underlying physics. This is remarkable since it provides the muchneeded quantitative understanding and lays the foundation for using these modes to measure machine properties as well as beam motion in phase space. In the following, we present this new approach to untangle the mixed SVD modes and the results obtained, focusing on the betatron modes due to ("in-plane") betatron oscillation, the coupling modes due to transverse coupling ("out-plane" betatron oscillation), and the dispersion modes due to synchrotron oscillation. All of these modes have been 
commonly observed, for example, at the storage ring of the Advanced Photon Source (APS) [12].

Although we will discuss primarily the coupled betatron modes, this paper deals with the quantitative understanding of the SVD modes in general rather than the analysis of transverse coupling. Coupling modes are discussed here as an illustration and one important application.

\section{ANALYSIS OF MIXED BETATRON MODES DUE TO COUPLING}

Let us start with the general description of beam orbits in a ring. Usually beam motion is dominated by the linear properties of a lattice. To a good approximation, a horizontal orbit can be described by

$$
x(s)=x_{0}(s)+x_{a}(s)+x_{b}(s)+D_{x}(s) \delta,
$$

where $x_{0}$ is the zeroth order orbit around which small oscillations occur due to betatron and synchrotron motions. Using action-angle variables $\left(J_{a}, \phi_{a}, J_{b}, \phi_{b}\right)$ for the decoupled transverse phase spaces (labeled with subscripts $a$ and $b$ ) and following the notations used in Refs. [15-17], the betatron oscillation due to the " $a$ " mode is given by

$$
x_{a}=\sqrt{2 J_{a} \bar{\beta}_{a}} \gamma \cos \left(\phi_{a}+\psi_{a}\right),
$$

where $\beta_{a}$ and $\psi_{a}$ are the beta function and phase advance of the $a$ mode, respectively, and $\gamma$ is the diagonal element in the coupling matrix $\bar{V}$ that relates the action-angle coordinates to the laboratory coordinates via $\left(x, x^{\prime}, y, y^{\prime}\right)^{T}=G^{-1} \bar{V}\left(\sqrt{2 J_{a}} \cos \phi_{a},-\sqrt{2 J_{a}} \sin \phi_{a},(a \rightarrow\right.$ b) $)^{T}$ with

$$
\bar{V}=\left(\begin{array}{cc}
\gamma I & \bar{C} \\
-\bar{C}^{\dagger} & \gamma I
\end{array}\right)
$$

where $†$ means symplectic conjugate, and

$$
G=\left(\begin{array}{cc}
G_{a} & 0 \\
0 & G_{b}
\end{array}\right), \quad G_{a, b}=\left(\begin{array}{cc}
\frac{1}{\sqrt{\beta_{a, b}}} & 0 \\
\frac{\alpha_{a, b}}{\sqrt{\beta_{a, b}}} & \sqrt{\beta_{a, b}}
\end{array}\right) .
$$

The betatron oscillation due to the " $b$ " mode is

$$
x_{b}=\sqrt{2 J_{b} \beta_{a}} c_{b} \cos \left(\phi_{b}+\psi_{b}+\Delta \psi_{b}\right),
$$

where $\psi_{b}$ is the phase advance of the $b$ mode, $c_{b}=$ $\sqrt{\bar{C}_{11}^{2}+\bar{C}_{12}^{2}}$, and $\Delta \psi_{b}=\arctan \left(\bar{C}_{12} / \bar{C}_{11}\right)$. The last term in Eq. (2) is coupled from the energy $\delta$ oscillation through the dispersion $D_{x}$. Vertical orbits can be similarly expressed by switching $x$ to $y$ and $a$ to $b$, and with $c_{a}=$ $\sqrt{\bar{C}_{22}^{2}+\bar{C}_{12}^{2}}$ and $\Delta \psi_{a}=-\arctan \left(\bar{C}_{12} / \bar{C}_{22}\right)$.

In a MIA analysis, a large set of beam orbits is recorded to form a data matrix $B$ (usually with the closed orbit subtracted). In order to understand the SVD modes of $B$, here we analytically construct a data matrix from the general beam motion described above and then carry out the SVD analysis analytically. For theoretical investigation, it is important to express $B$ in a proper matrix form (so-called physical-base decomposition) that separates the temporal phase-space variation and the spatial lattice responses. To simplify the discussion, we first consider the $x$ plane with no synchrotron oscillation and ignore BPM noise. With each row given by Eq. (2), we can write

$$
B=U V^{T}
$$

where

$$
U=\frac{1}{\sqrt{P}}\left(\begin{array}{ccc}
\cdots & \sqrt{2 J_{a}^{p}} \cos \phi_{a}^{p} & \cdots \\
\cdots & -\sqrt{2 J_{a}^{p}} \sin \phi_{a}^{p} & \cdots \\
\cdots & \sqrt{2 J_{b}^{p}} \cos \phi_{b}^{p} & \cdots \\
\cdots & -\sqrt{2 J_{b}^{p}} \sin \phi_{b}^{p} & \cdots
\end{array}\right)_{4 \times P}^{T},
$$

which is normalized by $\sqrt{P}$ for convenience, and

$$
V=\left(\begin{array}{cccc}
\vdots & \vdots & \vdots & \vdots \\
\sqrt{\beta_{a}^{m}}\left(\gamma^{m}, 0, c_{b}^{m}, 0\right) R\left(\psi_{a}^{m}, \psi_{b}^{m}\right. & \left.+\Delta \psi_{b}^{m}\right) \\
\vdots & \vdots & \vdots & \vdots
\end{array}\right)_{M \times 4} .
$$

Here each row of the $V$ matrix has four entries and is represented by the compact matrix form. The blockdiagonal rotation matrix $R$ propagates the motion described by $U$ in the (Floquet) phase space at a certain location to the $m$ th BPM. The $U$ matrix is determined by beam motion and could change from one data set to another, while the $V$ matrix is determined by a machine lattice and is usually unchanged. Given a measured matrix $B$, the goal is to recover $U$ and $V$ as much as possible in order to measure beam phase-space motion and/or to measure machine lattice functions.

To relate the physical modes with the four SVD modes due to coupled betatron oscillations, we first note that the action and angle distributions can generally be assumed independent and the angle is uniformly distributed, thus

$$
U^{T} U=\operatorname{diag}\left(\bar{J}_{a}, \bar{J}_{a}, \bar{J}_{b}, \bar{J}_{b}\right) \equiv \bar{J},
$$

where $\bar{J}_{a, b}=\left\langle J_{a, b}\right\rangle_{p}$, i.e., ensemble average of $J_{a, b}$. With proper normalization, we can turn this equation into an orthogonal condition and connect $U$ to $\hat{U}$ via [18]

$$
U=\hat{U} O \sqrt{\bar{J}} .
$$

Here $O$ is a $4 \times 4$ orthogonal matrix to be determined. The spatial vectors can be related via Eqs. (1), (7), and (11) as

$$
S \hat{V}^{T}=O \sqrt{\bar{J}} V^{T}
$$


The orthogonality of $\hat{V}$ requires

$$
S \hat{V}^{T} \hat{V} S=S^{2}=O \sqrt{\bar{J}} V^{T} V \sqrt{\bar{J}} O^{T} .
$$

We see that the orthogonal matrix $O$ must diagonalize the symmetric matrix $\sqrt{\bar{J}} V^{T} V \sqrt{\bar{J}}$ to yield the measured singular values. The existence of this Schur decomposition is guaranteed, but it could be hard to obtain analytical expressions for $O$ and the singular values $S$. Nonetheless, it is important to realize this structure [19], which is a key to untangling the SVD modes.

To appreciate this approach, note that we only need to diagonalize the matrix $\sqrt{\bar{J}} V^{T} V \sqrt{\bar{J}}$ in Eq. (13), which is $4 \times 4$ in this case. In comparison, the approach used in [14] deals with the covariance matrix $B^{T} B=V \bar{J} V^{T}$, which is much larger and thus much harder to solve.

Before continuing with the coupled case, let us examine how this approach works for the much simpler betatron motion in 1 degree of freedom. In this case, $\bar{J}$ is proportional to the identity matrix, $V_{m}=\left(\sqrt{\beta^{m}}, 0\right) R\left(\psi^{m}\right)$, and

$$
\sqrt{\bar{J}} V^{T} V \sqrt{\bar{J}}=\bar{J} \sum_{m} \beta\left(\begin{array}{cc}
\cos ^{2} \psi & \sin \psi \cos \psi \\
\sin \psi \cos \psi & \sin ^{2} \psi
\end{array}\right) .
$$

This matrix can be easily diagonalized by $O=R\left(\phi_{0}\right)$, a rotation with angle $\phi_{0}$ given by

$$
\phi_{0}=\frac{1}{2} \arctan \left(\frac{\sum_{m} \beta \sin 2 \psi}{\sum_{m} \beta \cos 2 \psi}\right) .
$$

Then Eq. (12) becomes

$$
S \hat{V}^{T}=\left(\begin{array}{ccc}
\cdots & \sqrt{\bar{J} \bar{\beta}_{m}} \cos \left(\psi^{m}-\phi_{0}\right) & \cdots \\
\cdots & \sqrt{\bar{J} \beta_{m}} \sin \left(\psi^{m}-\phi_{0}\right) & \cdots
\end{array}\right) .
$$

The phase advance can be determined up to a constant by

$$
\psi^{m}=\tan ^{-1}\left(\frac{\sigma_{2} \hat{V}_{2 m}}{\sigma_{1} \hat{V}_{1 m}}\right) .
$$

These are the same results obtained in [14].

Having convinced ourselves of the new approach, let us now continue with the coupling case. First note that the diagonal matrix $\bar{J}$ in Eq. (10) commutes with the blockdiagonal rotation matrix $R\left(\psi_{a}, \psi_{b}+\Delta \psi_{b}\right)$ in Eq. (9); thus the effect of $\sqrt{\bar{J}}$ in $\sqrt{\bar{J}} V^{T}$ of Eq. (12) is to scale $\gamma$ by $\sqrt{\bar{J}}_{a}$ and $c_{b}$ by $\sqrt{\bar{J}_{b}}$. Therefore, for $4 \mathrm{D}$ transverse motion, Eq. (12) can be written as

$S \hat{V}^{T}=O\left(\cdots, R^{T}\left(\psi_{a}, \psi_{b}+\Delta \psi_{b}\right)\left(\begin{array}{c}\sqrt{\overline{J_{a} \beta_{a}} \gamma} \\ 0 \\ \sqrt{\bar{J}_{b} \beta_{a}} c_{b} \\ 0\end{array}\right), \cdots\right)_{4 \times M}$,

where $O$ is determined by the diagonalization of

$$
\sum_{m} \beta_{a} R^{T}\left(\begin{array}{cccc}
\bar{J}_{a} \gamma^{2} & 0 & \sqrt{\bar{J}_{a} \bar{J}_{b}} \gamma c_{b} & 0 \\
0 & 0 & 0 & 0 \\
\bar{J}_{a} \bar{J}_{b} \gamma c_{b} & 0 & \bar{J}_{b} c_{b}^{2} & 0 \\
0 & 0 & 0 & 0
\end{array}\right) R=O^{T} S^{2} O
$$

Here $R$ stands for $R\left(\psi_{a}, \psi_{b}+\Delta \psi_{b}\right)$. The left-hand side consists of three independent submatrices of the shape

$$
\sum_{m} \beta_{a} g\left(\begin{array}{cc}
\cos \theta_{1} \cos \theta_{2} & \cos \theta_{1} \sin \theta_{2} \\
\sin \theta_{1} \cos \theta_{2} & \sin \theta_{1} \sin \theta_{2}
\end{array}\right),
$$

with $g=\bar{J}_{a} \gamma^{2}, \theta_{1}=\theta_{2}=\psi_{a}$ and $g=\bar{J}_{b} c_{b}^{2}, \theta_{1}=\theta_{2}=$ $\psi_{b}+\Delta \psi_{b}$ for the upper and lower diagonal blocks, respectively, and $g={\sqrt{\bar{J}} a \bar{J}_{b}}_{\gamma} c_{b}, \theta_{1}=\psi_{a}, \theta_{2}=\psi_{b}+\Delta \psi_{b}$ for the upper off-diagonal block. In rings with a large number of BPMs, the phase advances $\psi_{a, b}^{m}(\bmod 2 \pi)$ tend to be uncorrelated and uniformly distributed. Thus the off-diagonal submatrices average to small values if not zeroes.

When the off-diagonal blocks are zeroes, Eq. (19) reduces to two separate $2 \times 2$ matrix diagonalizations that are the same as in Eq. (14). Thus we have the solution $O=$ $R\left(\psi_{a}^{0}, \psi_{b}^{0}\right)$, a block-diagonal rotation with angles $\psi_{a}^{0}$ and $\psi_{b}^{0}$ to diagonalize the diagonal submatrices. The exact values of these angles are in fact unimportant. Now the SVD modes can be written explicitly as

$$
S \hat{V}^{T}=\left(\begin{array}{ccc}
\cdots & \sqrt{\bar{J}_{a} \bar{\beta}_{a}} \gamma \cos \left(\psi_{a}-\psi_{a}^{0}\right) & \cdots \\
\cdots & {\sqrt{\bar{J}_{a}} \beta_{a} \gamma \sin \left(\psi_{a}-\psi_{a}^{0}\right)}_{\cdots} \\
\cdots & {\sqrt{\bar{J}_{b} \beta_{a}} c_{b} \cos \left(\psi_{b}+\Delta \psi_{b}-\psi_{b}^{0}\right)}_{\cdots}^{\cdots} \\
\cdots & \sqrt{\bar{J}_{b} \beta_{a}} c_{b} \sin \left(\psi_{b}+\Delta \psi_{b}-\psi_{b}^{0}\right) & \cdots
\end{array}\right)_{4 \times M} .
$$

This is a simple and intuitive result. It naturally extends the betatron modes in Eq. (16) to accommodate coupling modes. The temporal components of these modes are obvious from Eq. (11), i.e.,

$$
\hat{U}=\frac{1}{\sqrt{P}}\left(\begin{array}{ccc}
\cdots & \sqrt{2 J_{a} / \bar{J}_{a}} \cos \left(\phi_{a}+\psi_{a}^{0}\right) & \cdots \\
\cdots & -\sqrt{2 J_{a} / \bar{J}_{a}} \sin \left(\phi_{a}+\psi_{a}^{0}\right) & \cdots \\
\cdots & \sqrt{2 J_{b} / \bar{J}_{b}} \cos \left(\phi_{b}+\psi_{b}^{0}\right) & \cdots \\
\cdots & -\sqrt{2 J_{b} / \bar{J}_{b}} \sin \left(\phi_{b}+\psi_{b}^{0}\right) & \cdots
\end{array}\right)_{4 \times P}^{T}
$$

The corresponding singular values are given by the norms of the above four spatial vectors, i.e., [20]

$$
\begin{gathered}
\sigma_{\beta_{1,2}}^{2}=\frac{M \bar{J}_{a}}{2}\left[\overline{\beta_{a} \gamma^{2}} \pm \sqrt{\left.{\overline{\beta_{a} \gamma^{2} \cos 2 \psi_{a}}}^{2}+{\overline{\beta_{a} \gamma^{2} \sin 2 \psi_{a}^{2}}}^{2}\right],}\right. \\
\sigma_{c_{1,2}}^{2}=\frac{M \bar{J}_{b}}{2}\left[\overline{\beta_{a} c_{b}^{2}} \pm \overline{\beta_{a} c_{b}^{2} \cos 2\left(\psi_{b}+\Delta \psi_{b}-\psi_{b}^{0}\right)}\right] .
\end{gathered}
$$

Here the subscript $\beta_{1,2}\left(c_{1,2}\right)$ indicates the first and second 
betatron (coupling) modes, and the overhead bar means averaging over the BPMs. Given a machine lattice, these expressions can be used to estimate the expected singular values of the coupled betatron modes.

\section{UNTANGLING MIXED MODES WITH A ROTATION}

When the off-diagonal submatrices do not average out, there is no simple analytical solution for Eq. (19). The left-hand side of Eqs. (21) and (22) have to be generalized to $O^{T} S \hat{V}$ and $\hat{U} O$, respectively. The matrix $O$ will contain rotations that mix the physical bases in $U$ and result in temporal vectors in $\hat{U}$ containing mixed tunes in their spectrum, according to Eq. (11). Fortunately, since we have understood the role of the matrix $O$, its analytical expression becomes less important. Instead it is critical to be able to numerically compute $O$ on the basis of a measured $B$. The recipe relies on finding an orthogonal matrix $O$ such that the Fourier spectrum of each temporal vector in $\hat{U} O$ shows a unique tune as suggested by Eq. (11).

If the mixing is small, the rotation $O$ should be close to the identity matrix $I$, and the problem can be linearized by taking $O=I+\sum \theta_{i j} L_{i j}$, where $\theta_{i j}$ 's are the rotation angles and $L_{i j}=\left(\delta_{i k} \delta_{j l}-\delta_{i l} \delta_{j k} ; k, l=1: 4\right)$ is the infinitesimal rotation matrix in the space spanned by the $i$ th and $j$ th vectors. Let $F_{j}[u]$ be the $j$ th Fourier component of the temporal vector $u$, then the $j$ th component of the $k$ th rotated vector $\tilde{u}_{k}$ is

$$
F_{j}\left[\tilde{u}_{k}\right]=F_{j}\left[u_{k}\right]+\sum_{\left\{\theta_{\alpha \beta}\right\}} F_{j}\left[u_{l}\right]\left(\delta_{\alpha l} \delta_{\beta k}-\delta_{\alpha k} \delta_{\beta l}\right) \theta_{\alpha \beta} .
$$

The coefficients $F_{j}\left[u_{k, l}\right]$ are known from the singular vectors. Collecting $F_{j}\left[\tilde{u}_{k}\right]=0$ for all $j$ 's and $k$ 's for which $F_{j}\left[\tilde{u}_{k}\right]$ needs to be minimized sets up a set of equations to solve for the rotation angles. In general, there are six angles in a four-dimensional rotation, two of them are the angles $\psi_{a, b}^{0}$ discussed above. Thus there are at most four angles that need to be determined through minimizing the "mixed-in" Fourier components. The minimization criteria could use peak values of the spectra.

In the case where the mixing is large, one may (a) extract an approximate solution of $\sqrt{\bar{J}} V^{T}$ by harmonic projection, i.e., compute the sine and cosine coefficients of the recorded turn-by-turn histories at the horizontal and vertical tunes; (b) compute $\tilde{O}=S \hat{V}^{T} \cdot(\sqrt{\overline{\bar{J}}})^{*} V^{T}$, where the quantities with an overhead tilde represent the approximate solutions and the * represents the series of matrix operations: transpose, pseudoinverse, then transpose again; (c) apply the above recipe for small mixing to $\hat{U} \tilde{O}$ and obtain a small correction $\tilde{\tilde{O}}$ to $\tilde{O}$; (d) combine them together to obtain $O=\tilde{O} \tilde{\tilde{O}}$. If neces- sary, the last two steps may be repeated. Once $O$ is determined, the physical modes can be obtained from the SVD modes via Eqs. (11) and (12). Note that if the betatron oscillation is a clean sinusoidal oscillation (as in ideal resonant excitations), the harmonic projection itself may yield the physical bases with good accuracy. However, when dealing with other than perfect excitations (e.g., with significant damping) or measuring some complicated phase-space evolution (in fact, whenever $U$ cannot be given as a priori), SVD modes and the above recipe should be useful.

An obvious application of the untangled coupling modes $O^{T} S \hat{V}^{T}$, i.e., the right-hand side of Eq. (21), is the measurement of local couplings (important particularly for high-energy colliders). For example, with data from both horizontal and vertical planes, all the phases $\psi_{a}, \psi_{b}, \Delta \psi_{a}$, and $\Delta \psi_{b}$ can be determined. Meanwhile, from the amplitudes of the betatron and coupling modes, $\beta_{a} \gamma^{2}, \beta_{b} \gamma^{2}, \beta_{a} c_{b}^{2}, \beta_{b} c_{a}^{2}$ can be determined up to a scaling constant. We will not go into the details here (available in [21]) since this paper is not focused on transverse coupling. It should also be noted that an understanding of the SVD modes is important not only for coupling measurement. There are other potential benefits as well, such as better measurement of beam phase-space evolution and better exploration of modes due to unknown physics by confidently subtracting known physical modes from the data.

To illustrate our understanding, a simulation was done using the APS ring lattice. Two skew quadrupoles were arbitrarily set to generate coupling (with maximum $\operatorname{det} C \simeq 0.014$ ). A particle with initial kick in both $x$ and $y$ was tracked 1000 turns using ELEGANT [22]. The first four SVD modes (others are much smaller) of horizontal data are shown in Fig. 1 where slight mixing is apparent in the Fourier spectra. Using the above algorithm, the rotation matrix $O$ was found to have the angles $\theta_{13}=$ $0.156, \theta_{14}=0.109, \theta_{23}=0.027$, and $\theta_{24}=-0.083$. After rotation, the spectra of $\hat{U} O$ and the vectors $O^{T} S \hat{V}^{T}$ are shown in Fig. 2 where the mixing is clearly removed. The residual mixed peaks around $120 \mathrm{kHz}$ are due to the aliased $2 \nu_{y}$ resonance, which can be removed as well and have little effect on the coupling modes. Figure 3 shows the amplitude of the coupling modes before and after the mixing is untangled. The dots are scaled $\sqrt{\beta_{a}} c_{b}$ from machine model for comparison. Clearly the untangled coupling modes yield the expected lattice function. Similar results were obtained for other lattice functions.

\section{MODE MIXING DUE TO DISPERSION}

After working through transverse coupling, it is easy to include synchrotron oscillation (as well as other oscillations with well-defined spectra) in the analysis. For the physical bases $U$ and $V$, we need to add the energy 

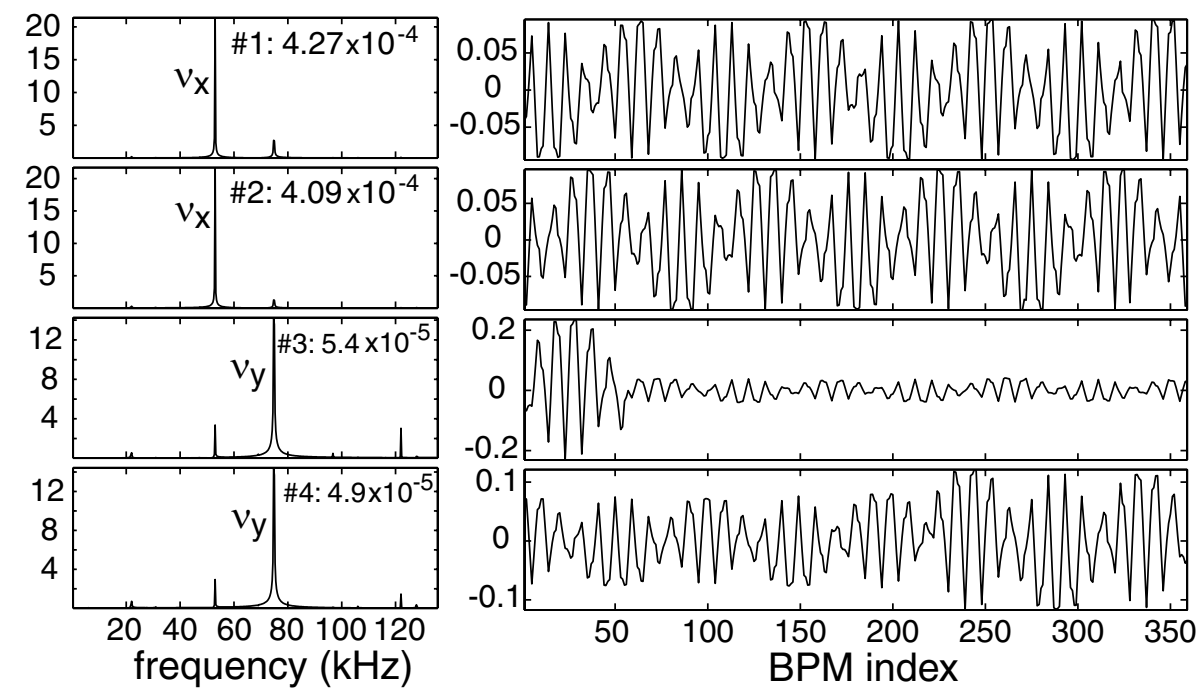

FIG. 1. Fourier spectra of the temporal vectors (left) and the spatial vectors (right) of the SVD modes. Mode numbers and singular values are shown in the upper right corners.

oscillation $\delta$ and dispersion $D_{x}$, respectively. Since the energy oscillation is independent of betatron oscillation, Eq. (10) still holds by adding a new diagonal element $\sigma_{\delta}^{2}$, the rms energy oscillation of the beam centroid (not the energy spread of the beam). Furthermore, the orthogonality condition in Eq. (19) needs to be extended by adding

$$
\sum_{m}\left(\begin{array}{c}
\sqrt{\bar{J}_{a}} \sigma_{\delta} D_{x} \sqrt{\beta_{a}} \gamma \cos \psi_{a} \\
\sqrt{\bar{J}_{a}} \sigma_{\delta} D_{x} \sqrt{\beta_{a}} \gamma \sin \psi_{a} \\
\sqrt{\bar{J}_{b}} \sigma_{\delta} D_{x} \sqrt{\beta_{a}} c_{b} \cos \left(\psi_{b}+\Delta \psi_{b}\right) \\
\sqrt{\bar{J}_{b}} \sigma_{\delta} D_{x} \sqrt{\beta_{a}} c_{b} \sin \left(\psi_{b}+\Delta \psi_{b}\right) \\
\sigma_{\delta}^{2} D_{x}^{2}
\end{array}\right)
$$

and its transpose as a new column and row. Again, the off-diagonal elements tend to average to zeroes for a ring with a large number of BPMs. In such a case, the spatial vectors in Eq. (21) will be extended by a dispersion vector $\sigma_{\delta} D_{x}$ whose corresponding temporal vector is the centroid energy oscillation $\delta / \sqrt{P} \sigma_{\delta}$. In cases where the offdiagonal terms do not average out, mixing will occur and one has to untangle the SVD modes with an extended $O$ matrix using the given recipe.

\section{EXPERIMENTAL EXAMPLE}

Here we present an experimental example to further confirm our analysis. The data were taken from the storage ring of the Advanced Photon Source. The beam initially had a steady-state betatron oscillation due to a single-bunch instability and was excited longitudinally
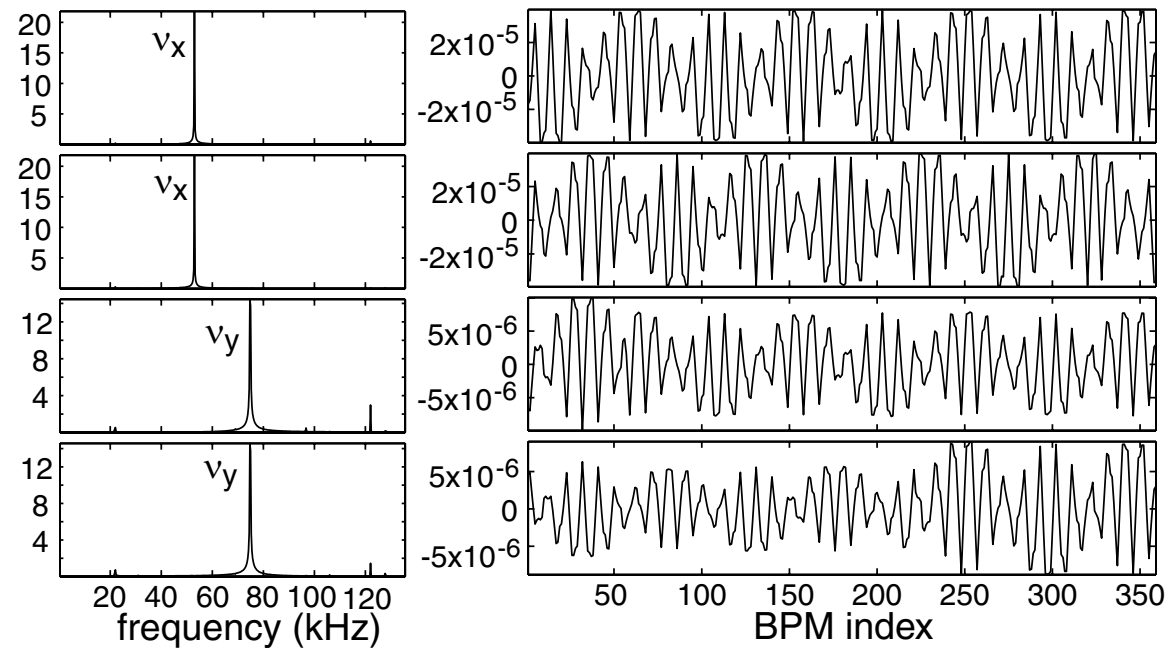

FIG. 2. Modes of Fig. 1 after a rotation to untangle mixing. 


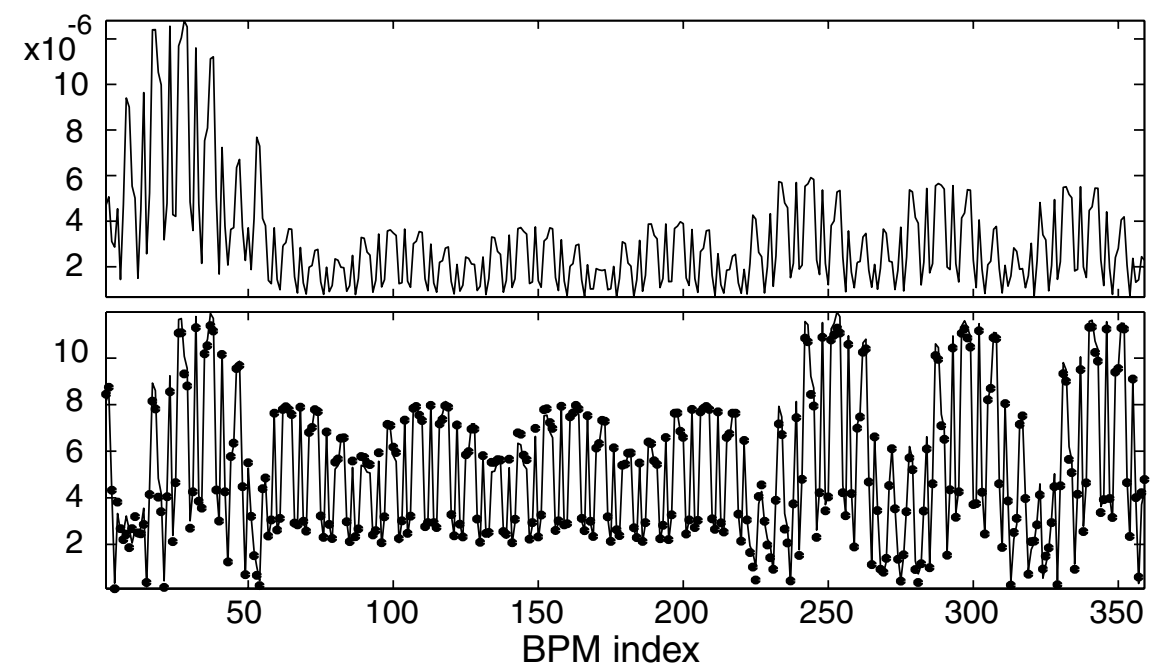

FIG. 3. Amplitudes of the spatial coupling vectors before (top) and after (bottom) the rotation. Dots are the scaled lattice function $\sqrt{\beta_{a}} c_{b}$.

using an rf phase kick. Turn-by-turn data were collected at all BPMs after the kick. The beam history at one BPM is shown in Fig. 4.

There are 186 usable BPMs in the data set. SVD analysis yields three significant modes. The first four singular values are 14.0, 9.0, 7.9, and 0.8, respectively. The spectra of the first three temporal vectors are shown on the left in Fig. 5. We see that the synchrotron and betatron modes are very well separated, although a little mixing is visible. After a small rotation of angles $\theta_{12}=-0.040$ and $\theta_{13}=$ 0.0035 , the mixing is untangled as can be seen in the spectra of the rotated temporal vectors on the right in Fig. 5. As explained above, the mixing tends to be larger if the number of BPMs is smaller. We randomly selected 50 out of the 186 BPMs and did an SVD analysis of them. The first four singular values became $15.4,9.1,7.5$, and

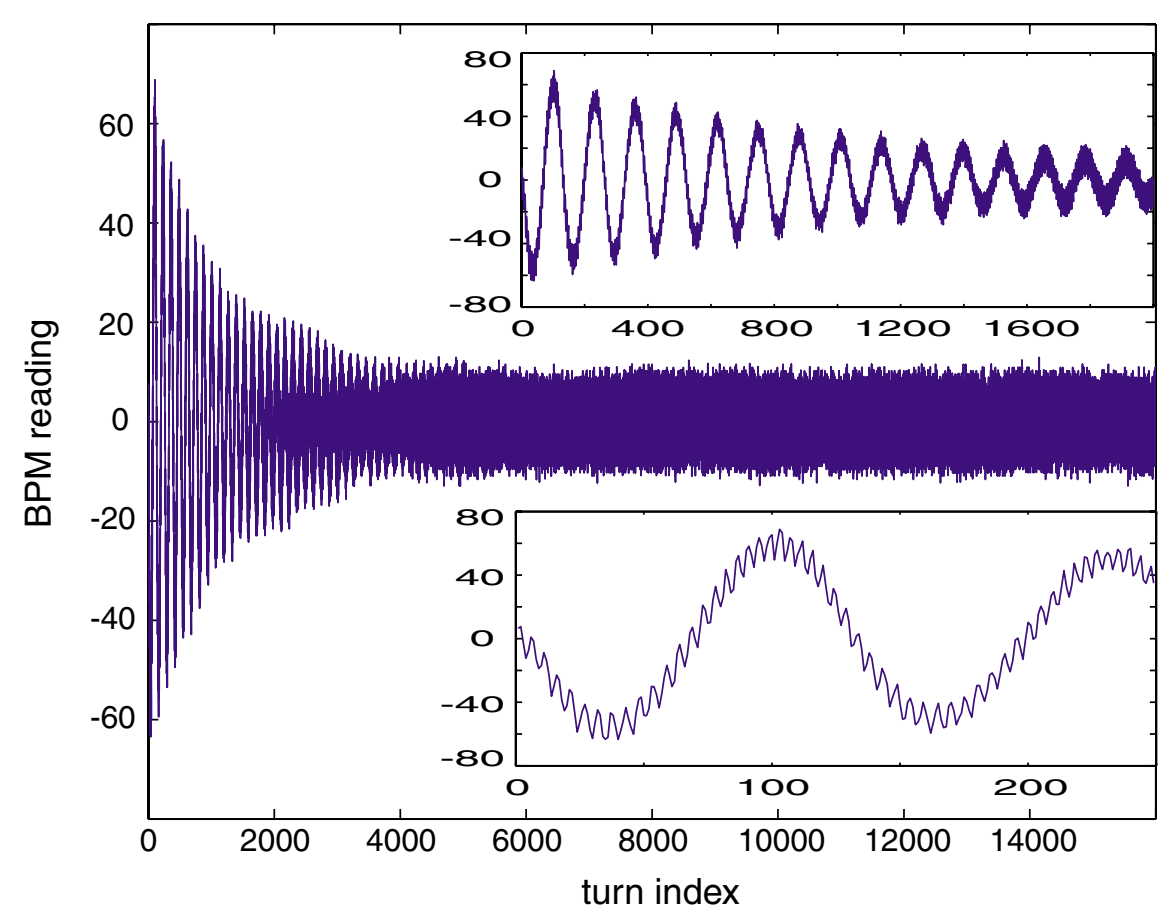

FIG. 4. (Color) Beam history at one BPM. The upper insert is the first 2000 turns showing synchrotron oscillation due to a longitudinal phase kick. The lower insert is the first 250 turns showing a betatron oscillation (due to instability) superimposed on the synchrotron oscillation. 

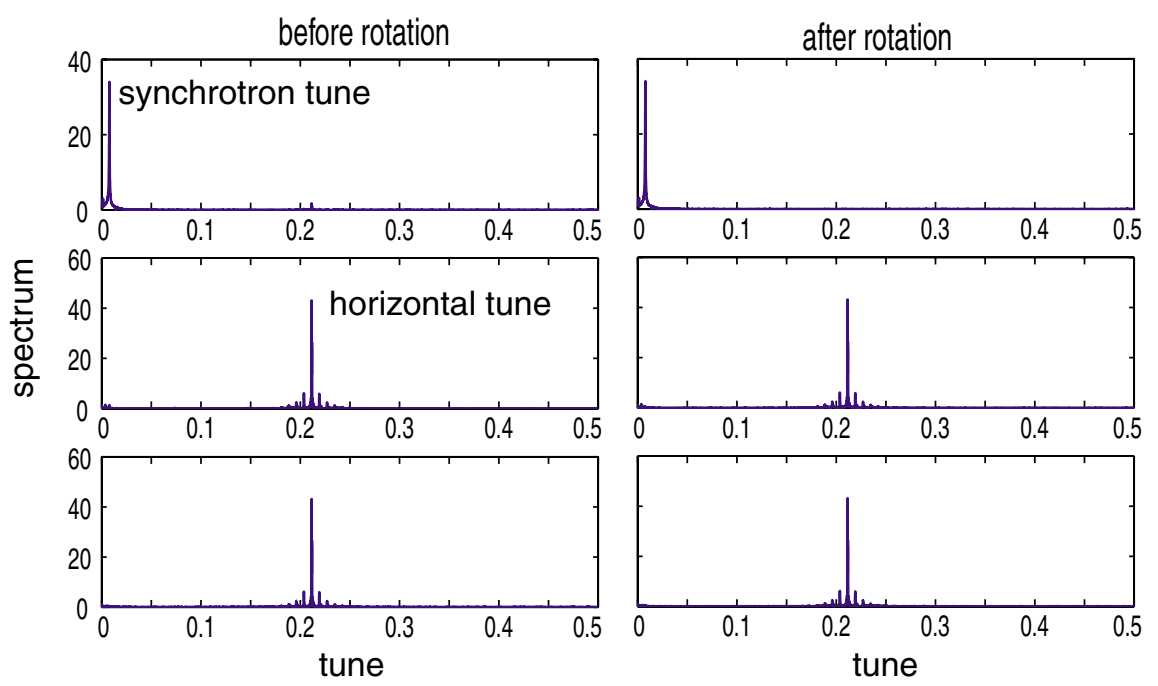

FIG. 5. (Color) The spectra of the first three modes using 186 BPMs. The spectra of the SVD modes are on the left and there is a little mode mixing. The spectra of the rotated SVD modes are on the right.

1.5 , respectively. Note that the fourth singular value almost doubles, i.e., the noise floor is much higher due to the smaller number of BPMs. Again, the spectra of the first three temporal vectors are shown on the left in Fig. 6. As expected, now the mode mixing becomes quite significant, which again can be untangled by a rotation of angles $\theta_{12}=-0.12$ and $\theta_{13}=-0.25$ as shown on the right in Fig. 6.

To show the importance of using untangled modes, we computed the beta function using the spatial vectors of the mixed SVD modes and the rotated spatial vectors for both cases (using 186 BPMs and 50 BPMs). The results are plotted in Fig. 7, which shows only a small section of the ring for clarity. We see that the beta functions from the untangled modes using 186 BPMs (blue dots) and using 50 BPMs (green circles) agree with each other very well. The beta function from the SVD modes using 186 BPMs (black crosses) is fairly close to the untangled ones since the mixing is small. However, the beta function from the SVD modes using 50 BPMs (red crosses) is obviously wrong since the mode mixing is significant, as shown above.

This simple example experimentally confirmed our analysis of the mixed SVD modes and the technique to untangle the mixing by rotating the singular vectors. It also illustrates the importance of untangling the mixed modes, especially when the number of BPMs is small.
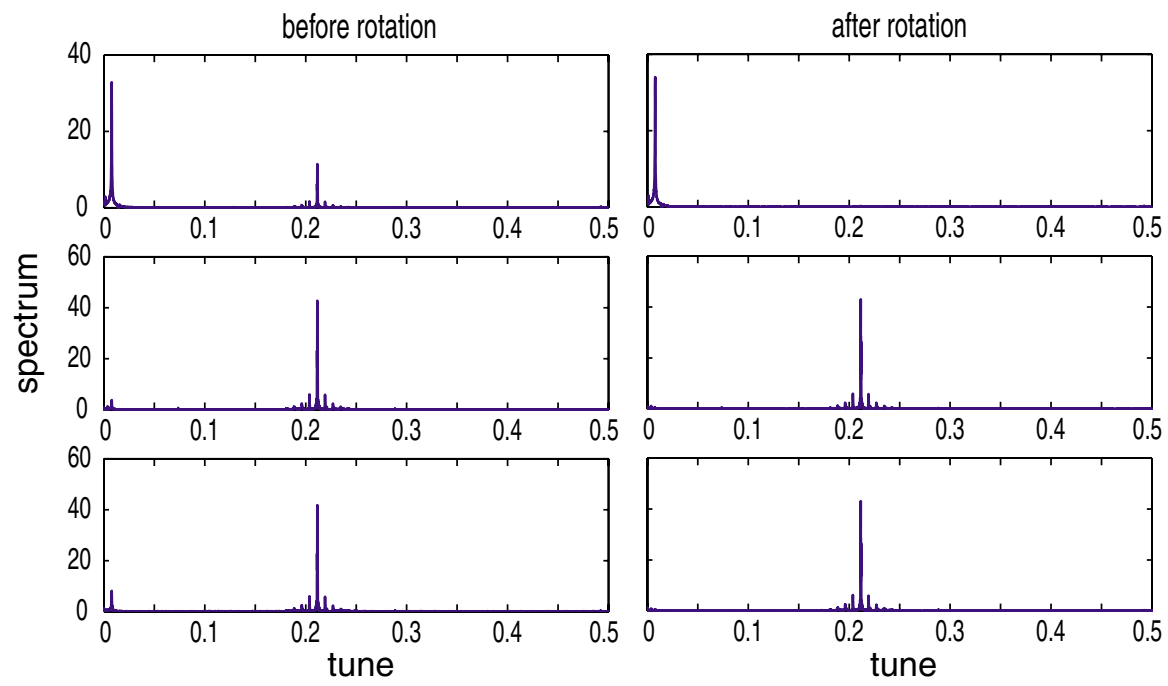

FIG. 6. (Color) Similar to Fig. 5 but using 50 instead 186 BPMs. Now the mode mixing is significant in the SVD modes but is apparently removed by a rotation. 


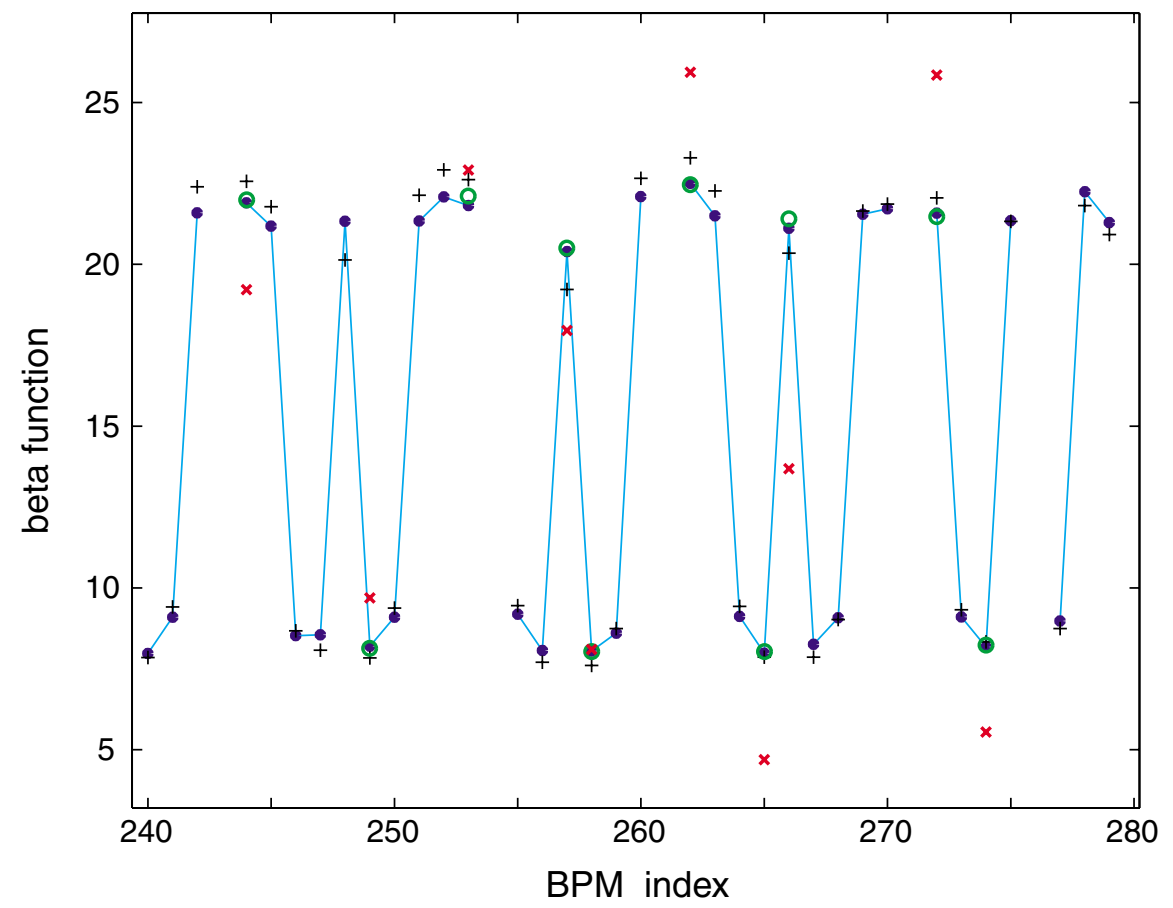

FIG. 7. (Color) Comparison of beta functions measured using SVD modes and rotated SVD modes. Black crosses are from SVD modes using 186 BPMs, blue dots are from rotated modes using 186 BPMs, red crosses are from SVD modes using 50 BPMs, and green circles are from rotated modes using 50 BPMs.

\section{CONCLUDING REMARKS}

To conclude, we presented a simple approach to understand and untangle the orthogonal SVD modes used in model-independent analysis and established the muchneeded quantitative relationship between these modes and machine properties. In particular, the coupled betatron modes were analyzed in detail to demonstrate this approach.

After submission of this manuscript, I learned that the independent component analysis technique [23-25] developed in other fields may also be used to resolve mode mixing on the basis of statistical independence of the signals.

\section{ACKNOWLEDGMENTS}

This work was supported by the U.S. Department of Energy, Office of Basic Energy Sciences, under Contract No. W-31-109-ENG-38. I am grateful to W. Guo and K. Harkay for providing the experimental data used in the example. Thanks are due to M. Borland, L. Emery, V. Sajaev, and Y. Chae for their help in using ELEGANT for the simulation. I would also like to thank L.C. Teng for discussion and K. Harkay for her support.

[1] J. Irwin, C.-x. Wang, Y.T. Yan, K. L. F. Bane, Y. Cai, F.-J. Decker, M. G. Minty, G.V. Stupakov, and F. Zimmermann, Phys. Rev. Lett. 82, 1684 (1999).
[2] Chun-xi Wang, Ph.D. thesis, Stanford University, 1999; also Report No. SLAC-R-547, 2003.

[3] R. Gnanadesikan, Methods for Statistical Data Analysis of Multivariate Observations (Wiley, New York, 1997), 2nd ed.

[4] T.W. Anderson, An Introduction to Multivariate Statistical Analysis (Wiley, New York, 1984), 2nd ed.

[5] M. Seidel, DESY Internal Note, 1999.

[6] J. Irwin and Y.T. Yan, in Proceedings of the 2000 European Particle Accelerator Conference, Vienna (Austrian Academy of Sciences, Vienna, 2000), p. 151.

[7] Y. Cai, J. Irwin, M. Sullivan, and Y.T. Yan, in Proceedings of the 2001 Particle Accelerator Conference, Chicago, IL (IEEE, Piscataway, NJ, 2001), p. 3555 .

[8] P. Castro, C. Lackas, M. Seidel, and M. Wendt, in Proceedings of the 2002 European Particle Accelerator Conference, Paris (EPS-IGA, Geneva, 2002), p. 503.

[9] A. Wolski, M. C. Ross, M. D. Woodley, and J. Nelson, in Proceedings of the 2002 European Particle Accelerator Conference, Paris (Ref. [8]), p. 1205.

[10] Y. Yan, Y. Cai, J. Irwin, and M. Sullivan, Report No. SLAC-PUB-9368, 2002.

[11] Chun-xi Wang, in Proceedings of the 2003 Particle Accelerator Conference, Portland, OR (IEEE, New York, 2003), p. 3407.

[12] Chun-xi Wang, in Proceedings of the 2003 Particle Accelerator Conference, Portland, OR (Ref. [11]), p. 3410.

[13] L. Emery, Proceedings of the 2003 Particle Accelerator Conference, Portland, OR (Ref. [11]), p. 3464. 
[14] Chun-xi Wang, Vadim Sajaev, and Chih-Yuan Yao, Phys. Rev. ST Accel. Beams 6, 104001 (2003).

[15] A.W. Chao and M. Tigner, Handbook of Accelerator Physics and Engineering (World Scientific, River Edge, NJ, 1998).

[16] D. Sagan and D. Rubin, Phys. Rev. ST Accel. Beams 2, 074001 (1999).

[17] D. A. Edwards and L. C. Teng, IEEE Trans. Nucl. Sci. 20, 885 (1973). Note that we list this reference for its historical significance, but the notations we used are different.

[18] Note that here $\hat{U}$ and $\hat{V}$ contain the four coupled betatron modes, i.e., we are using $\hat{U}$ and $\hat{V}$ from a "thin" SVD with the null space vectors ignored. See Gene H. Golub and Charles F. Van Loan, Matrix Computations (Johns Hopkins University, Baltimore, MD, 1996), 3rd ed., p. 451.

[19] For example, an immediate conclusion can be drawn from Eq. (12) since orthogonal transformations preserve the amplitude (2-norm)

$$
\beta_{a}\left(\bar{J}_{a} \gamma+\bar{J}_{b} c_{b}\right)=\sum_{i=1}^{4}\left(\sigma_{i} \hat{V}_{i}\right)^{2}
$$

which is an extension of the beta function expression in Ref. [14] to include coupling effects.

[20] By eliminating $\psi_{b}^{0}$ and $\Delta \psi_{b}$, the $\sigma_{c_{1,2}}^{2}$ can be expressed with readily available lattice functions as

$$
\sigma_{c_{1,2}}^{2}=\frac{M \bar{J}_{b}}{2}\left[\overline{\beta_{a} c_{b}^{2}} \pm \sqrt{A^{2}+B^{2}}\right],
$$

where

$$
\begin{aligned}
& A=\overline{\left(\bar{C}_{11}^{2}-\bar{C}_{12}^{2}\right) \beta_{a} \cos 2 \psi_{b}}-2 \overline{\bar{C}_{11} \bar{C}_{12} \beta_{a} \sin 2 \psi_{b}}, \\
& B=\overline{\left(\bar{C}_{11}^{2}-\bar{C}_{12}^{2}\right) \beta_{a} \sin 2 \psi_{b}}+2 \overline{\bar{C}_{11} \bar{C}_{12} \beta_{a} \cos 2 \psi_{b}} .
\end{aligned}
$$

[21] Chun-xi Wang and Rama Calaga, in Proceedings of the 2004 European Particle Accelerator Conference, Lucerne, Switzerland, http://accelconf.web.cern.ch/accelconf/e04/default.htm, p. 1470.

[22] M. Borland, Advanced Photon Source, Light Source Note No. LS-287, 2000.

[23] W. Guo (private communication).

[24] See, for example, Aapo Hyvärinen, Juha Karhunen, and Erkki Oja, Independent Component Analysis (Wiley, New York, 2001).

[25] L. Molgedey and H. G. Schuster, Phys. Rev. Lett. 72, 3634 (1994). 